\title{
CORRECTION OF MOBILE MAPPING TRAJECTORIES IN GNSS-DENIED ENVIRONMENTS USING AERIAL NADIR AND AERIAL OBLIQUE IMAGES
}

\author{
P. Fanta-Jende ${ }^{1 *}$, F. Nex ${ }^{1}$, M. Gerke ${ }^{2}$, J. Lijnen ${ }^{1}$, G. Vosselman ${ }^{1}$ \\ ${ }^{1}$ Dept. of Earth Observation Science, Faculty ITC, University of Twente, The Netherlands - \\ (p.l.h.jende, f.nex, j.j.lijnen, george.vosselman)@utwente.nl \\ ${ }^{2}$ Institute of Geodesy and Photogrammetry, Technische Universität Braunschweig, Germany - \\ m.gerke@tu-bs.de
}

EuroCow-M3DMaN

KEY WORDS: Image orientation, Mobile Mapping, Aerial Oblique Images, Aerial Nadir Images

\begin{abstract}
:
Mobile mapping enables highly accurate as well as high-resolution image data capture at low cost and high speed. As a terrestrial acquisition technique predominately employed in urban, and thus built-up areas, non-line-of-sight and multipath effects challenge its absolute positioning capabilities provided by GNSS. In conjunction with IMU drift, the platform's trajectory has an unknown accuracy, which influences the quality of the data product. By employing a highly accurate co-registration technique for identifying tie correspondences between mobile mapping images and aerial nadir as well as aerial oblique images, reliable ground control can be introduced into an adjustment solution. We exemplify the performance of our registration results by showcasing adjusted mobile mapping trajectories in four different test areas, each with about 100 consecutive recording locations (approx. $500 \mathrm{~m}$ length) in the city centre of Rotterdam, The Netherlands. The mobile mapping data has been adjusted in different configurations, i.e. with nadir or oblique aerial correspondences only and if possible in conjunction. To compare the horizontal as well as the vertical accuracy before and after the respective adjustments, more than 30 ground control points were surveyed for these experiments. In general, the aim of our technique is not only to correct mobile mapping trajectories in an automated fashion but also to verify their accuracy without the need to acquire ground control points. In most of our test cases, the overall accuracy of the mobile mapping image positions in the trajectory could be improved. Depending on the test area, an RMSE in 3D between 15 and $21 \mathrm{~cm}$ and an RMSE in 2D between 11 and $18 \mathrm{~cm}$ is achievable.
\end{abstract}

\section{INTRODUCTION}

As a terrestrial geo-data acquisition platform, mobile mapping $(\mathrm{MM})$ is affected by GNSS-induced positioning issues, such as multipath or non-line-of-sight effects. Consequently, the absolute position of the platform cannot be reliably determined at all times and acquired data postings' accuracy is unknown and likely impaired. A correction of the platform's trajectory or alternatively the data product is usually conducted by introducing external references, such as ground control points (GCPs) or digital map data. Whereas GCPs offer a high accuracy but are labour-intensive to acquire and to integrate, maps are generalisations of the real world, difficult to intersect with acquired MM data, and cannot necessarily provide for surveying-grade accuracy. In our previous work, we have presented co-registration approaches for mobile mapping and aerial nadir and oblique images (Fanta-Jende et al., 2019; Jende et al., 2018a; Jende et al., 2018b). Airborne platforms are not affected by the aforementioned GNSS issues, and aerial images can thus be used as a reference. The major challenge is to overcome the large perspective differences to identify mutual features in the terrestrial and aerial data set. Nadir and oblique aerial images have different properties as well; hence, two different co-registration approaches with the mobile mapping images have been devised. Both approaches will be briefly discussed in the methodology section (3.1 and 3.2). After the identification of correspondences between the terrestrial and aerial data set, multiple adjustment options are possible. For the experiments in this paper, only tie points that are at least visible in two aerial images have been used for data adjustment.

\section{RELATED WORK}

Approaches to improve terrestrial data are manifold in their characteristics. For instance, distinctions can be made on the real-time or post-processing property of an approach, whether the correction procedure relies on improving the platform's data directly or utilises external data as a reference.

In our case, comparable procedures, i.e. relying on the introduction of an external reference for data adjustment, have been developed by various authors. Cheng et al. (2015) introduce a hierarchical registration approach between mobile and aerial laser scanning point clouds relying on the extraction of building contours and road features. Although no GCPs have been used to evaluate the absolute accuracy of the result, the relative mean accuracy between both data sets after registration is around half a metre in the vertical and horizontal dimension. Gruen et al. (2013) present an approach to combine UAV with MM point clouds for the generation of complete 3D representations. Therefore, both point clouds have been merged using manually measured control points. The relative accuracy between both data sets has been assessed by check points and reaches an RMSE of $11 \mathrm{~cm}$ horizontally and $20 \mathrm{~cm}$ in height. Similarly, Molina et al. (2017) developed a tightly-coupled positioning system for the simultaneous acquisition of UAV and ground-based images. The UAV is following an optical target installed on the roof of the terrestrial platform during the acquisition. Depending on the configuration, either the UAV's positioning is used for the correction of the terrestrial platform or the other way around. Planimetric as well as a vertical accuracy below $10 \mathrm{~cm}$ is well achievable with this concept.

\footnotetext{
* Corresponding author
} 
Tournaire et al. (2006) suggest utilising zebra crossings for the registration of MM stereo data and aerial imagery. To this end, zebra crossings are extracted with a knowledge-based template approach. Although a thorough statistical setup is missing, the authors claim to have reached sub-decimetre accuracy.

Ji et al. (2015) use correspondences between aerial ortho images and MM images for the estimation of the MM platform's trajectory. Since the search for correspondences is per se noisy and returns many false positives, the authors employ a particle filter solution to estimate the optimal trajectory. The authors report a horizontal accuracy of about $1.0 \mathrm{~m}$ and a vertical accuracy of about $1.30 \mathrm{~m}$ if areas without valid correspondences are truncated.

Hussnain et al. (2018) developed an approach to correct mobile laser scanning platform trajectories by the introduction of correspondences to aerial nadir images. By integrating these correspondences as well as IMU readings into a B-spline estimation, the adjusted trajectories achieve an accuracy (RMSE) of $9 \mathrm{~cm}$ in $\mathrm{X}, 14 \mathrm{~cm}$ in $\mathrm{Y}$, and $14 \mathrm{~cm}$ in Z.

Javanmardi et al. (2017) also utilise correspondences between mobile laser scanning and aerial images to correct the terrestrial data set. The authors report an average error of $11 \mathrm{~cm}$.

In our previous work (Jende et al., 2018a), we analysed the improvement of MM images compared to surveyed GCPs in three test areas using solely correspondences to aerial nadir images. Although a planimetric accuracy of up to $6 \mathrm{~cm}$ has been reached, in some case, an error in height has been introduced due to a very high altitude of the aerial platform (4500 metres). This led to glancing intersections due to a bad intersection geometry and consequently an uncertainty in height.

In this paper, correspondences to aerial oblique and nadir images are used standalone and in conjunction with each other to correct the MM platform's trajectory.

\section{METHODOLOGY}

As mentioned earlier, the major challenge of employing aerial images as a reference data set for MM trajectory correction is a reliable co-registration technique. In our case, MM images are encoded in an equirectangular projection covering 360 degrees horizontally and 180 degrees vertically. This projection entails severe distortions compared to an (aerial) perspective image. Hence, MM images need to be remapped to increase the resemblance and thus the geometric similarity to the aerial images to simplify or rather enable a reliable registration of the data sets. As aerial nadir and oblique images depict a scene from different angles, the perspective remapping has to account for these individual properties. To this end, two different coregistration techniques, one between $\mathrm{MM}$ and aerial nadir images and one between MM and aerial oblique images, have been developed.

\subsection{Co-registration of aerial nadir and mobile mapping images}

Aerial nadir images feature a top-down view on the scene. Hence, common entities that are visible in both, the aerial and MM data set, are mostly roads. To this end, MM images are quasi-ortho projected by assuming a ground plane directly located beneath the platform (see Figure 1).

The registration is based on corner detection in conjunction with phase-correlation. Since the data sets are not necessarily perfectly aligned but are displaced within an expected maximum error, orientation parameters are used to constrain the search space for correspondences. With a very high inlier rate of more than $95 \%$, this technique proved to be successful. For more information, please see Jende et al. (2018a).

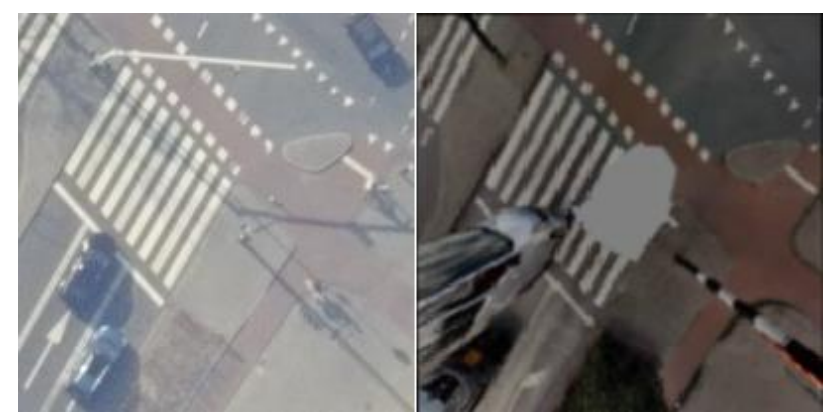

Figure 1. Left: Aerial nadir image, right: re-projected MM image

\subsection{Co-registration of aerial oblique and mobile mapping images}

The ground is a mutual geometric surface in object space of aerial nadir and MM images. While the ground is easy to detect or rather assumed and to be utilised as a projection surface for the registration scenario earlier, this relationship cannot be exploited directly for aerial oblique and MM images.

Building façades and other vertical planar structures along the road, however, are visible in the aerial oblique as well as MM image data set. Since the MM images are linked for data adjustment using visual odometry, a sparse point cloud can be generated from these correspondences. Façades can now be detected by a highly constrained plane fitting approach. Extruding inlying sparse points on a façade to a patch allows for the discretisation of an artificial surface in object space. These patches are used to extract image information of MM as well as aerial oblique images (see Figure 2).
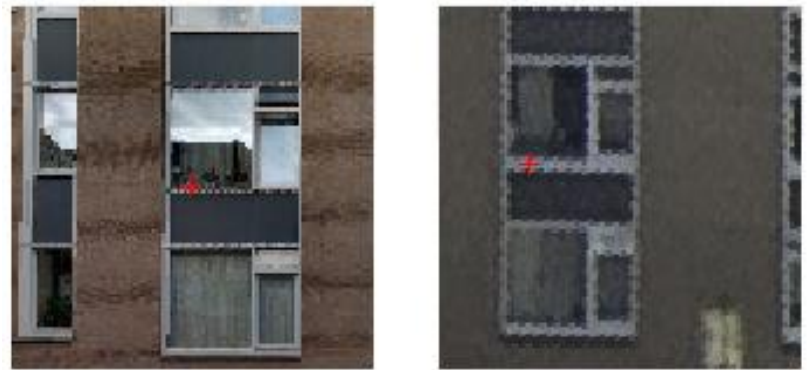

Figure 2. Left: Mobile mapping image patch, right: aerial oblique image patch

Both patches are registered using mutual information. The inlier rate of this technique is about $80 \%$. For further details on this approach, please see Fanta-Jende et al. (2019); Jende et al. (2018b)

\subsection{Mobile mapping data adjustment}

Both co-registration pipelines use projection surfaces to register MM with aerial images. Although the matching is performed locally on geometrically modified images, the transformations are known and the correspondences can be translated into their original geometry.

In order to adjust MM images that do not have direct correspondences to the aerial images, MM images are linked with each other using a visual odometry/structure from motion approach. Although a classical bundle adjustment by locking 


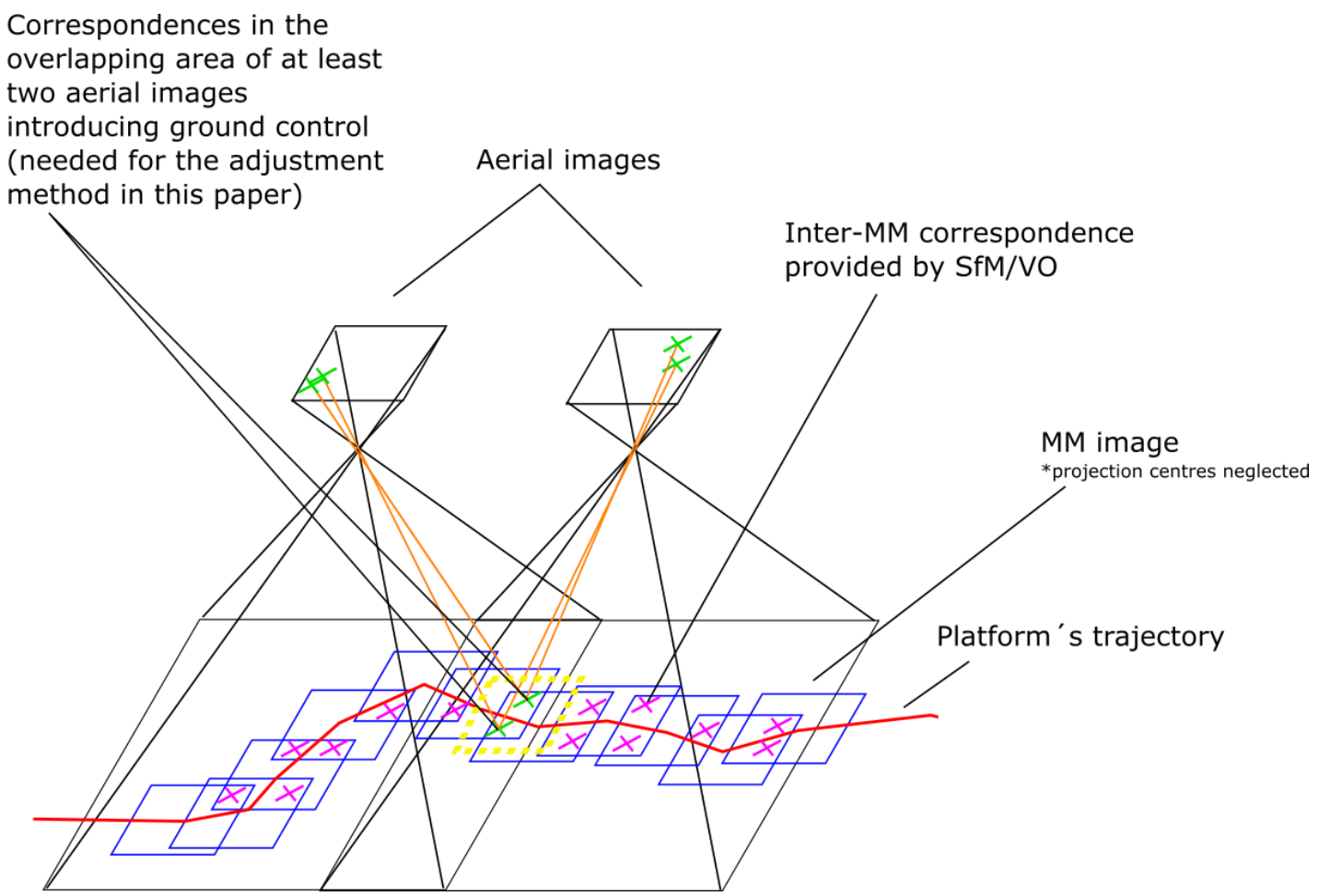

Figure 3. Schematics of the selected adjustment method

the exterior orientation of the aerial cameras while the MM recording locations are to be adjusted is possible, the experiments in this paper are based on an adjustment where the image observations of the aerial images are triangulated and used as GCPs. Therefore, only correspondences, which are visible in at least two aerial images, are used (see Figure 3). The GCPs are all weighted with $10 \mathrm{~cm}$ standard deviation, corresponding to 1 pixel in average in the aerial nadir and oblique image. Future experiments will focus on different and more flexible adjustment scenarios also with respect to the weighting strategy.

\section{EXPERIMENTS}

To ascertain the performance of our registration approaches in different scenarios, four trajectories in Rotterdam, The Netherlands, have been selected. The aerial nadir images in this experiment were acquired at an altitude of $4470 \mathrm{~m}$ and have a ground sampling distance of about $10 \mathrm{~cm}$. Similarly to the experiments conducted in our previous publication (Jende et al., 2018a), this may lead to an uncertainty in height. The aerial oblique images were acquired in a pentacam-fashion at an altitude of $450 \mathrm{~m}$ and have a ground sampling distance ranging from 5 to $15 \mathrm{~cm}$. The test areas vary in their characteristics ranging from open to narrow roads or surrounded by buildings on both or only one side (see Figure 4).

Table 1 gives an overview on the test areas. For area 3 (blue trajectory in Figure 4), only oblique correspondences were used, as the road does not have any salient road markings, hence almost no reliable correspondences to the aerial nadir images could be identified and were thus discarded. Similarly, area 4 (yellow trajectory in Figure 4) only features façades that are partially occluded by vegetation or relatively far away from the platform's trajectory. This impedes the plane fitting and the reprojection process, which are pivotal to create image patches from both data sets. Thus, only a few correspondences to the aerial oblique images were returned and were discarded for these experiments. In total, 34 GCPs are used as check points to assess the accuracy of the adjusted recording locations.

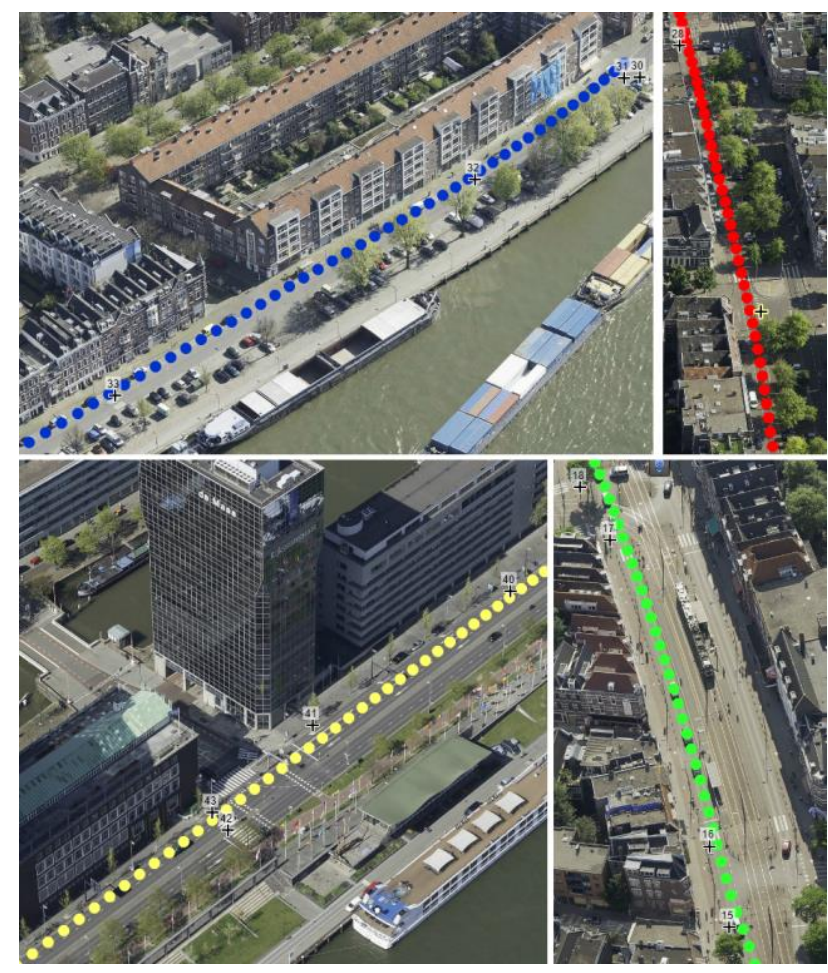

Figure 4. Characteristics of the four test areas (only subsets). Area 1 (green trajectory), area 2 (red traj.), area 3 (blue traj.), area 4 (yellow traj.). The recording locations and surveyed GCPs in the selected subset have been projected into an overlapping aerial oblique image. 


\begin{tabular}{|l|cc|c|c|c|}
\hline Area & \multicolumn{2}{|c|}{$\begin{array}{c}\text { Number of tie } \\
\text { points }\end{array}$} & $\begin{array}{c}\text { Number } \\
\text { of MM } \\
\text { images }\end{array}$ & $\begin{array}{c}\text { Joint } \\
\text { adjustment }\end{array}$ & $\begin{array}{c}\text { Number } \\
\text { check } \\
\text { points }\end{array}$ \\
\cline { 2 - 3 } & Nadir & Oblique & & & \\
\hline 1 & 81 & 68 & 140 & yes & 11 \\
2 & 26 & 29 & 87 & yes & 4 \\
3 & 0 & 95 & 88 & no & 8 \\
4 & 92 & 0 & 150 & no & 11 \\
\hline
\end{tabular}

Table 1. Overview of the four test areas

4.1 Adjustment results using correspondences to the aerial nadir images

Area 1, 2, and 4 can be adjusted with nadir correspondences only.

\begin{tabular}{|l|l|l|l|l|l|l|}
\hline Area & \multicolumn{2}{|c|}{ RMSE X } & \multicolumn{2}{c|}{ RMSE Y } & \multicolumn{2}{c|}{ RMSE Z } \\
\hline & before & after & before & after & before & after \\
\hline 1 & 0.14 & $\mathbf{0 . 1 2}$ & 0.12 & $\mathbf{0 . 1 1}$ & 0.23 & $\mathbf{0 . 2 1}$ \\
\hline 2 & $\mathbf{0 . 1 5}$ & 0.21 & $\mathbf{0 . 5 2}$ & 0.53 & $\mathbf{0 . 1 9}$ & 0.23 \\
\hline 4 & 0.19 & 0.19 & 0.16 & $\mathbf{0 . 1 4}$ & $\mathbf{0 . 2 0}$ & 0.24 \\
\hline
\end{tabular}

Table 2. Adjustment result using only correspondences to the aerial nadir images [in metres]. Best result in bold.

As mentioned earlier, glancing intersections due to the high altitude of the aircraft may have a negative impact on the vertical component after the adjustment. This effect introduced a larger error in height in the area 2 and 4.

In the case of area 2, utilising only correspondences to the aerial nadir data set had an overall negative impact on the accuracy of the trajectory. First, area 2 has a lower accuracy than the other trajectories and secondly the unequal distribution of correspondences may have had an impact on the adjustment (see Figure 5).

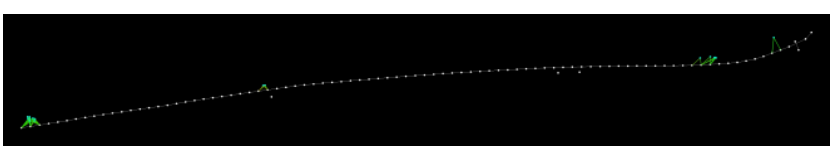

Figure 5. Distribution of correspondences (green) of area 2 along the trajectory (white); [rotated by 90 degrees]

Area 4 only shows marginal updates after the adjustment. However, some check points along the trajectory ascertain a more significant update especially in the Y direction (see Table 3). Designing an experiment with different weights for the correspondences to the aerial images may better explain this behaviour.

\begin{tabular}{|l|l|l|l|l|}
\hline & \multicolumn{2}{|c|}{ CP 39 } & \multicolumn{2}{c|}{ CP 43 } \\
\hline & before & after & before & after \\
\hline $\mathrm{X}$ & $\mathbf{0 . 0 2}$ & 0.06 & 0.15 & $\mathbf{0 . 1 4}$ \\
\hline $\mathrm{Y}$ & $\mathbf{- 0 . 1 0}$ & -0.15 & -0.21 & $\mathbf{- 0 . 1 2}$ \\
\hline $\mathrm{Z}$ & 0.22 & $\mathbf{0 . 1 7}$ & $\mathbf{0 . 0 7}$ & 0.12 \\
\hline
\end{tabular}

Table 3. Exemplary trajectory updates at two check points in area 4 . Best result in bold [in metres].

Area 1 shows minor improvements in all three dimensions and decreases the RMSE in 3D by almost $2 \mathrm{~cm}$ (see Table 8). Although this update is marginal, it exemplifies that the registration procedure can be also utilised to verify the accuracy of mobile mapping trajectories by determining the magnitude of an update.
4.2 Adjustment results using correspondences to the aerial oblique images

Similar to the previous section, three test areas could be processed with using only correspondences to the aerial oblique images.

\begin{tabular}{|l|l|l|l|l|l|l|}
\hline \multirow{2}{*}{ Area } & \multicolumn{2}{|c|}{ RMSE X } & \multicolumn{2}{c|}{ RMSE Y } & \multicolumn{2}{c|}{ RMSE Z } \\
\cline { 2 - 7 } & before & after & before & after & before & after \\
\hline 1 & 0.14 & $\mathbf{0 . 1 3}$ & $\mathbf{0 . 1 2}$ & 0.14 & 0.23 & 0.23 \\
\hline 2 & $\mathbf{0 . 1 5}$ & 0.21 & 0.52 & $\mathbf{0 . 1 3}$ & $\mathbf{0 . 1 9}$ & 0.28 \\
\hline 3 & $\mathbf{0 . 1 2}$ & 0.12 & 0.24 & $\mathbf{0 . 2 2}$ & $\mathbf{0 . 1 9}$ & 0.20 \\
\hline
\end{tabular}

Table 4. Adjustment result using only correspondences to the aerial oblique images [in metres]. Best result in bold.

Since the tie correspondences are weighted with $10 \mathrm{~cm}$ standard deviation in the adjustment and the statistics of the differences in all dimensions before and after the update are very low, area 1 shows no significant improvement (see Table 5).

\begin{tabular}{|l|l|l|l|}
\hline & X & Y & Z \\
\hline Mean & 0.003 & -0.006 & 0.001 \\
\hline Std. dev. & 0.019 & 0.018 & 0.011 \\
\hline Max & 0.038 & 0.027 & 0.054 \\
\hline Min & -0.044 & -0.056 & -0.040 \\
\hline
\end{tabular}

Table 5. Statistics of the differences before and after the adjustment of area 1 with correspondences to the aerial oblique images [in metres].

Area 2, however, has been updated in all three dimensions. Whereas the previous experiment using only correspondences to the aerial nadir images worsened the accuracy in every dimension, utilising aerial oblique images improved the accuracy in Y dramatically. Overall, the combined RMSE in 3D has been improved by more than $10 \mathrm{~cm}$ (from 0.33 to 0.22 , see Table 8).

Most correspondences to the aerial oblique data set could be identified in area 3. Although there were 95 individual triangulated tie points, all the correspondences were identified on one side of the trajectory only due to the river on the other side of the road and thus the lack of any vertical structure to derive image patches (see Figure 1 (blue trajectory) and Figure $6)$. This property had some impact on the statistics of the adjustment, as the trajectory has been pulled by a couple of centimetres into a positive $\mathrm{X}$ and $\mathrm{Y}$ direction (see Table 6). The accuracy, however, only improved marginally.

\begin{tabular}{|l|l|l|l|}
\hline & $\mathrm{X}$ & $\mathrm{Y}$ & $\mathrm{Z}$ \\
\hline Mean & 0.017 & 0.027 & 0.013 \\
\hline Std. dev. & 0.016 & 0.017 & 0.022 \\
\hline Max & 0.042 & 0.057 & 0.041 \\
\hline Min & -0.032 & -0.007 & -0.028 \\
\hline
\end{tabular}

Table 6. Statistics of the differences before and after the adjustment of area 3 with correspondences to the aerial oblique images [in metres].

\subsection{Adjustment results using correspondences to the aerial oblique and nadir images}

In two test areas (1 and 2), a joint adjustment was possible. To this end, the correspondences to both, the aerial nadir and oblique data set, were fed into the adjustment. This increased the number of correspondences significantly (see Table 1). 


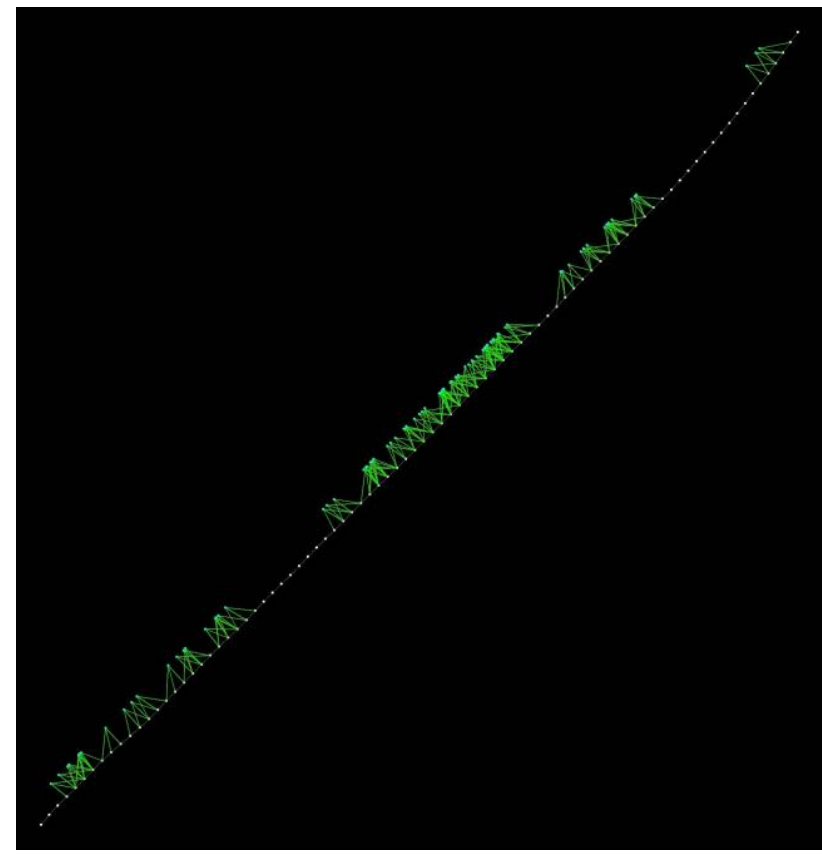

Figure 6. Distribution of correspondences (green) of area 3 along the trajectory (white). All the correspondences were identified on one side of the road.

\begin{tabular}{|l|l|l|l|l|l|l|}
\hline \multirow{2}{*}{ Area } & \multicolumn{2}{|c|}{ RMSE X } & \multicolumn{2}{c|}{ RMSE Y } & \multicolumn{2}{c|}{ RMSE Z } \\
\cline { 2 - 7 } & before & after & before & after & before & after \\
\hline 1 & 0.14 & $\mathbf{0 . 1 3}$ & 0.12 & $\mathbf{0 . 0 9}$ & 0.23 & $\mathbf{0 . 2 0}$ \\
\hline 2 & $\mathbf{0 . 1 5}$ & 0.18 & 0.52 & $\mathbf{0 . 1 8}$ & $\mathbf{0 . 1 9}$ & 0.27 \\
\hline
\end{tabular}

Table 7. Adjustment result using correspondences to the aerial oblique and nadir images [in metres]. Best result in bold.

Area 1 could be improved in every dimension, in $\mathrm{Y}$ even to a sub-decimetre level. This is an interesting finding, as an improvement to this extent is only enabled by the combination of both nadir and oblique image correspondences.

Area 2, however, does not necessarily benefit from a joint adjustment. Although the results have improved in $\mathrm{X}$ and $\mathrm{Y}$ in comparison to using only correspondences to the aerial nadir images, the accuracy in $\mathrm{Z}$ has been worsened.

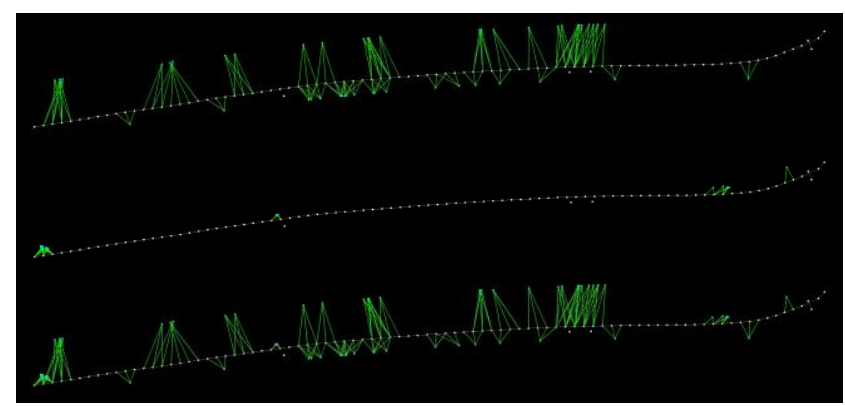

Figure 7. Distribution of correspondences in area 1. From top to bottom: aerial oblique correspondences, aerial nadir correspondences, and both combined.

Interestingly, the accuracy in $\mathrm{X}$ is higher than in the single-use cases. Figure 7 depicts the correspondences for area 2. As mentioned in the previous section (4.1), the nadir correspondences in area 2 are unequally distributed and cluttered at the left hand side of the trajectory. Hence, a joint adjustment is not necessarily averaging the results obtained from a single aerial nadir or aerial oblique result. It rather depends on the number, the distribution, and the individual properties of the tie points.

\subsection{Summary and discussion}

The previous sections compared different adjustment scenarios utilising correspondences to aerial nadir images, aerial oblique images, or both. Table 8 and Table 9 give an overview on the overall RMSE 2D and 3D values before and after the different adjustments.

\begin{tabular}{|l|c|c|c|c|}
\hline Area & \multirow{2}{*}{$\begin{array}{l}\text { RMSE } \\
\text { 2D before }\end{array}$} & \multicolumn{3}{|c|}{ RMSE 2D after } \\
\cline { 3 - 5 } & 0.13 & Nadir & Oblique & Combined \\
\hline 1 & 0.38 & 0.11 & 0.13 & $\mathbf{0 . 1 1}$ \\
\hline 2 & 0.19 & n/a & $\mathbf{0 . 1 8}$ & $\mathbf{0 . 1 8}$ \\
\hline 3 & 0.172 & $\mathbf{0 . 1 6 7}$ & n/a & n/a \\
\hline 4 & &
\end{tabular}

Table 8. RMSE combined in X, Y before and after respective adjustments. Best result in bold [in metres]. Borderline cases not rounded.

\begin{tabular}{|l|c|c|c|c|}
\hline \multirow{2}{*}{ Area } & \multirow{2}{*}{ RMSE } & \multicolumn{3}{|c|}{ RMSE 3D after } \\
\cline { 3 - 5 } & 3D before & Nadir & Oblique & Combined \\
\hline 1 & 0.17 & 0.15 & 0.17 & $\mathbf{0 . 1 5}$ \\
\hline 2 & 0.33 & 0.36 & 0.22 & $\mathbf{0 . 2 1}$ \\
\hline 3 & 0.190 & n/a & $\mathbf{0 . 1 8 6}$ & n/a \\
\hline 4 & $\mathbf{0 . 1 8}$ & 0.19 & n/a & n/a \\
\hline
\end{tabular}

Table 9 RMSE combined in X, Y, Z before and after respective adjustments. Best result in bold [in metres]. Borderline cases not rounded.

In general, the updates are all minor as the original recording locations already had a high accuracy. Area 2 is an exception where for instance the trajectory could be improved by almost up to $40 \mathrm{~cm}$ ( $\mathrm{Y}$ dimension in area 2 with aerial oblique correspondences only, see Table 4). This ascertains the feasibility of our approach to act on deviations of the trajectory and improve or also maintain the data's accuracy up to a low decimetre level. This effect is specifically visible with respect to the horizontal accuracy (Table 8) which could be improved for every test area.

In particular, using correspondences to the aerial nadir images for trajectory correction is certainly a viable option as long as the height component is neglected or weighted accordingly. Interestingly, the correspondences to the oblique images could also improve or at least verify the data's accuracy, although the complexity of the aerial oblique registration pipeline is comparatively high and is more prone to outliers than the nadir registration pipeline. Future efforts are directed towards the integration of the aerial images into the bundle adjustment. Additionally, the weights ought to be set in accordance to the expected measurement accuracy while an outlier removal mechanism will avoid a worsening of the accuracy.

\section{CONCLUSION}

This paper presented experimental results of an adjustment pipeline to correct mobile mapping image positions in a potentially GNSS-denied environment. Not only is the accuracy of the mobile mapping platform unknown but it may also be inaccurate. By yielding highly accurate correspondences between mobile mapping images and aerial nadir as well as aerial oblique images, the latter enable to act as a reference data set. To this end, image observations in the aerial data set have 
been triangulated and used as ground control points within a bundle adjustment. A comparison to surveyed ground control points was conducted to determine the horizontal as well as the vertical accuracy of the mobile mapping data before and after the adjustment. It could be shown that our procedure is able to verify and improve the trajectory.

\section{ACKNOWLEDGEMENTS}

This work is part of the TTW-Open Technology Programme with the project number 13589, which is financed by the Netherlands Organisation for Scientific Research (NWO). We would like to thank CycloMedia Technology B.V. for providing the mobile mapping data and supporting the analysis of the results.

\section{REFERENCES}

Cheng, L., Wu, Y., Tong, L., Chen, Y., Li, M., 2015. Hierarchical Registration Method for Airborne and Vehicle LiDAR Point Cloud. Remote Sensing 7 (10), 13921-13944.

Fanta-Jende, P., Nex, F., Gerke, M., Vosselman, G., 2019. CoRegistration Of Panoramic Mobile Mapping Images And Oblique Aerial Images. The Photogrammetric Record (accepted, forthcoming)

Gruen, A., Huang, X., Qin, R., Du, T., Fang, W., Boavida, J., Oliveira, A., 2013. Joint processing of UAV imagery and terrestrial mobile mapping system data for very high resolution city modeling. Int. Arch. Photogramm. Remote Sens. Spat. Inf. Sci, XL-1/W2, 175-182.

Hussnain, Z., Oude Elberink, S., Vosselman, G., 2018. An Automatic Procedure For Mobile Laser Scanning Platform 6dof Trajectory Adjustment. Int. Arch. Photogramm. Remote Sens. Spatial Inf. Sci., XLII-1, 203-209.

Javanmardi, M., Javanmardi, E., Gu, Y., Kamijo, S., 2017. Towards High-Definition 3D Urban Mapping: Road FeatureBased Registration of Mobile Mapping Systems and Aerial Imagery. Remote Sensing 9 (10), 975.

Jende, P., Nex, F., Gerke, M., Vosselman, G., 2018a. A fully automatic approach to register mobile mapping and airborne imagery to support the correction of platform trajectories in GNSS-denied urban areas. ISPRS Journal of Photogrammetry and Remote Sensing, 141, 86-99.

Jende, P., Nex, F., Gerke, M., Vosselman, G., 2018b. A Guided Registration Strategy Employing Virtual Planes To Overcome Non-Standard Geometries - Using The Example Of Mobile Mapping And Aerial Oblique Imagery. Int. Arch. Photogramm. Remote Sens. Spatial Inf. Sci.. XLII-2, 471-477.

Ji, S., Shi, Y., Shan, J., Shao, X., Shi, Z., Yuan, X., Yang, P., Wu, W., Tang, H., Shibasaki, R., 2015. Particle filtering methods for georeferencing panoramic image sequence in complex urban scenes. ISPRS Journal of Photogrammetry and Remote Sensing, 105, 1-12.

Molina, P., Blázquez, M., Cucci, D., Colomina, I., 2017. First Results of a Tandem Terrestrial-Unmanned Aerial mapKITE System with Kinematic Ground Control Points for Corridor Mapping. Remote Sensing 9(1), 60.
Tournaire, O., Soheilian, B., Paparoditis, N., 2006. Towards a Sub-Decimetric Georeferencing of Ground-Based Mobile Mapping Systems in Urban Areas: Matching Ground-Based and Aerial-based Imagery Using Roadmarks. ISPRS Commission I Symposium "From Sensors to Imagery". 